\title{
Deploying clustered wireless sensor network by multi-robot system
}

\begin{abstract}
Deployment strategy is vital for efficiency in applications of wireless sensor network. Deploying wireless sensor network in vast area is very hard and time consuming job. In many applications there is also need to deploy sink other than wireless sensor nodes which sink deployment is another issue. In this paper, we use spanning tree for autonomous deploying wireless nodes and sinks. Deploying actor in this method is multi-robot system. In this method robots act as dispenser for wireless nodes. Using spanning tree as exploration algorithm helps robot to full coverage deployment of nodes. For analyzing the algorithm using multi-robot a simulation test bed is developed with a parameterized environment with obstacle. The result shows performance of sensing coverage in different environments.
\end{abstract}

Keyword: WSN; Sink; Deployment; Multi-robot; Coverage 